\title{
Globe
}

Revue internationale d'études québécoises

\section{Diane Lamoureux : L'amère patrie. Féminisme et nationalisme dans le Québec contemporain. Montréal, Éditions du remue-ménage, 2001}

\section{Jacinthe Michaud}

Volume 5, numéro 2, 2002

URI : https://id.erudit.org/iderudit/1000683ar

DOI : https://doi.org/10.7202/1000683ar

Aller au sommaire du numéro

Éditeur(s)

Globe, Revue internationale d'études québécoises

ISSN

1481-5869 (imprimé)

1923-8231 (numérique)

Découvrir la revue

Citer ce compte rendu

Michaud, J. (2002). Compte rendu de [Diane Lamoureux : L'amère patrie Féminisme et nationalisme dans le Québec contemporain. Montréal, Éditions du remue-ménage, 2001]. Globe, 5(2), 171-175. https://doi.org/10.7202/1000683ar d'utilisation que vous pouvez consulter en ligne.

https://apropos.erudit.org/fr/usagers/politique-dutilisation/ 


\section{Recensions}

\section{Diane Lamoureux}

L'amère patrie. Féminisme et nationalisme

dans le Québec contemporain.

Montréal, Éditions du remue-ménage, 2001.

Après le référendum de 1995 , les débats sur le nationalisme et ses rapports avec les notions de 'nation, d'ethnicité et de citoyenneté ont rarement pris en considération l'articulation théorique avec le féminisme. C'est du moins ce qui était affirmé dans l'introduction de l'ouvrage collectif Malaises identitaires : échanges fêministes autour d'un Québec incertain, recueil d'articles que Diane Lamoureux avait dirigé en compagnie de Chantal Maillé et de Micheline de Sève en 1999. Avec L'amère patrie : féminisme et nationalisme dans le Québec contemporain, Diane Lamoureux se donne pour objectif de revisiter cette thématique avec davantage de perspective historique que dans ses écrits précédents.

Car ce n'est pas la première fois que Lamoureux exploite les thèmes du nationalisme et du féminisme, questions encore peu explorées par les féministes québécoises et canadiennes ${ }^{1}$. Son dernier ouvrage comprend trois parties. Dans la première, l'auteure effectue un rappel des idées politiques qui ont présidé à la naissance d'une conception moderne des notions de souveraineté, de citoyenneté et de nation. Nous ne pouvons que saluer l'expertise avec laquelle Lamoureux détaille les pensées philosophiques, depuis Bodin, Hobbes et Rousseau (théoriciens de l'État et de sa dynamique unificatrice), jusqu'aux conceptions de la citoyenneté et de son processus d'inclusion/exclusion des politiques et pratiques citoyennes de l'État providence. Le développement de l'idée

1. Voir surtout * Nationalism and Feminism in Québec: An Impossible Attraction ", Heather Jon Maroney et Meg Luxton (éd.), Feminism and Political Economy: Women's Work, Women's Struggles, Toronto, Methuen, 1987, p. 51-68.

Michel Lacroix [éd.], « Recensions ", Globe. Revue internationale d'études québécoises, vol. 5, $\mathrm{n}^{\circ} 2,2002$. 


\section{REVUE INTERNATIONALE D'ÉTUDES QUÉBÉCOISES}

de nation donne l'occasion à Lamoureux d'aborder le rôle réservé aux femmes à travers la maternité patriotique mise à contribution en temps de guerre et lors de la transmission des valeurs collectives.

La deuxième partie fait l'exposé du projet de modernisation de l'État québécois et du processus de formation identitaire qui, en raison de ses constantes métamorphoses, demeure inachevé. Le nationalisme, ici, est présenté comme étant le principal vecteur de cette modernisation. Mais dans la mesure où la démonstration de Lamoureux demeure résolument centrée sur la société québécoise, il devient difficile d'évaluer en quoi cette modernisation diffère dans d'autres segments de la société canadienne, elle-même non exempte de nationalisme et de mouvance identitaire. Pareillement, Lamoureux pose un regard sur la société civile essentiellement en fonction de l'action structurante de l'État providence. En cela, elle adopte une approche différente de celle développée par d'autres auteures, Jane Jenson entre autres, laquelle a développé la notion d' " univers du discours politique ${ }^{2}$ "où les acteurs collectifs légitimement reconnus (l'État providence étant à la fois un acteur et une force sélective de légitimation - le Québec ne fait pas exception à la règle dans ce cas) s'engagent dans un processus d'échange, de confrontation et de compromis avec pour résultat la constitution de "nouveaux paradigmes sociétaux ". Dans sa démarche, Lamoureux s'en tient à une vision régulatrice et structurante de l'État providence, ce qui n'est pas sans affecter sa conception du rôle réservé au féminisme dans la troisième et dernière partie de l'ouvrage.

Celle-ci comprend les deux derniers chapitres: "Féminisme et modernisation des femmes " et "Les formes de l'institutionnalisation partielle du féminisme . Tous deux forment ce qui doit être la thèse centrale et l'aboutissement de l'ouvrage. L'auteure y expose la convergence du projet nationaliste et de la modernisation du mouvement des femmes. On ne peut que déplorer cependant que l'analyse de cette convergence ne se fasse qu'en relation avec l'État et avec le nationalisme tel que promu par cet État. On n'y étudie que rarement la situation d'échange et de confrontation entre le mouvement des femmes et les autres composantes

2. Jane Jenson, "Paradigms and Political Discourse: Protective Legislation in France and the United States Before 1914!*, Revue canadienne de science politique, vol. 22, $\mathrm{n}^{\circ} 2,1989$, p. 235-258; « Gender and Reproduction : Or, Babies and the State ", Studies in political Economy, vol. 20, 1986, p. 9-46. 


\section{RECENSIONS}

de la société civile, également porteuses d'une vision nationaliste de la société québécoise, mais dans des cadres qui ne peuvent être entièrement assimilables à celui de l'État providence. Une approche moins régulatrice aurait permis à l'auteure de sortir d'un schéma linéaire où, " en parallèle avec le processus de construction "nationale", s'est profilé un mouvement de "modernisation" des femmes " (p. 137). Sur le plan théorique, il aurait été intéressant de voir comment le féminisme et le nationalisme évoluent autrement qu'en parallèle mais se transforment l'un et l'autre par une dynamique d'interruption et d'échange de signification.

Ainsi, dans "Féminisme et modernisation des femmes ", lorsque Lamoureux pose la question de la convergence du nationalisme et du féminisme, elle soulève en fait le problème de la double allégeance du mouvement des femmes : allégeance à la cause des femmes et allégeance à la cause de la libération nationale. Pareil dilemme a déjà été soulevé ailleurs chez les théoriciennes féministes de la post-colonialité ${ }^{3}$. La création d'un État national, selon Lamoureux, passe par la modernisation du Québec, et l'inscription de revendications féministes dans la législation québécoise participe de cette modernisation. Après une description des réalisations importantes dans ce domaine, comme l'égalité juridique, l'accès aux professions, à l'éducation, l'auteure nous remet en mémoire les lacunes qui restent à combler. Par ailleurs, elle explique que, si un nombre significatif de féministes ont pu apporter leur soutien à la construction d'un État providence au Québec, c'est sans doute parce que cet État a su ménager un certain accès à l'espace public et à la citoyenneté. Mais comment ne pas s'étonner quand Lamoureux trouve des a avantages non négligeables " au patriarcat public par opposition au patriarcat privé ? D'abord parce qu'il impose une distance entre le patriarcat et les rapports affectifs et interrompt ainsi le paternalisme, ensuite parce qu'il permet la politisation de la vie privée (p. 148). L'auteure aurait pu proposer quelques exemples probants à l'appui de sa thèse et s'efforcer de ne pas ignorer les formes nouvelles de régulation morale

3. Marie-Aimée Hélie Lucas, "Bound by the Family Code *, dans Miranda Davies (êd.), Third World - Second Sex, Londres, Zed Books, 1987, p. 3-15; Gayatri Chakavorty Spivak, - French Feminism Revisited: Ethics and Politics ", Judith Butler et Joan Scott (éd.), Feminist Theorize the Political, New York et Londres, Routledge, 1992, p. 54-85; Minh-ha Trinh T., Woman Native Other, Bloomintgton et Indianapolis, Indiana University Press, 1989. 


\section{REVUE INTERNATIONALE D'ÉTUDES QUÉBÉCOISES}

particulièrement intrusives dans la vie privée de plusieurs catégories sociales de femmes, dont les assistées sociales ne sont pas les moindres.

Dans le chapitre intitulé "Les formes de l'institutionnalisation partielle du féminisme ", Lamoureux nous propose une réflexion sur la place qu'occupe le féminisme dans le projet souverainiste, cherchant ainsi à savoir pourquoi certains segments du mouvement des femmes sont parties prenantes de l'institution alors que d'autres s'y refusent totalement (p. 153). Ce chapitre est malheureusement trop fragmenté, les liens entre les sections n'étant pas toujours évidents. L'auteure procède d'abord au rappel des trois grands courants théoriques qui ont eu quelque influence au sein du féminisme québécois et démontre le décalage entre théorie et pratique. On pourra déplorer le caractère schématique de cette démonstration, qui n'est soutenue par aucun exemple, en ce qui concerne le courant marxiste. Suivent quelques réflexions sur la politisation de l'identité que Lamoureux introduit par une lecture critique de deux auteurs, Ti-Grace Atkinson et Brian Walker, ce qui lui permet d'élaborer à la fois sur la construction de l'identité moderne et sur la façon dont le féminisme a analysé ce construit. Elle affirme que le mouvement féministe s'est constitué de l'intérieur et le précise en ces termes : "Le mouvement permet la constitution du sujet politique et, à certaines périodes, il peut même s'enferrer dans un solipsisme tout à fait narcissique. Sa cohérence ne lui est pas tant apportée de l'extérieur par un principe d'opposition que par le travail interne d'homogénéisation qu'il accomplit sur lui-même" (p. 167). Nulle influence n'est accordée aux questionnements portés par d'autres luttes sociales, comme les luttes anti-racistes par exemple, dont il est désormais reconnu qu'elles ont structuré considérablement le mouvement des femmes. Ici encore, le Québec n'est pas une exception.

Au terme de cet ouvrage, nous ne pouvons qu'abonder dans le sens d'une des thèses mises en valeur par Lamoureux. Le processus de modernisation de la société québécoise a permis l'émergence d'un nationalisme fort peu réfractaire au féminisme tel qu'il s'est développé dans le Québec contemporain. Différent en cela de nombreuses autres formes discursives de nationalisme, malgré quelques dérives verbales, celui qui a eu cours au Québec a su intégrer une vision du rôle des femmes qui ne les limite pas à la maternité (p. 17). La conceptualisation théorique que Lamoureux développe à propos des diverses tendances perceptibles dans le mouvement des femmes vaut la peine qu'on s'y arrête. Que nous 
soyons en accord ou non avec la reconstruction qu'elle propose du mouvement des femmes et de son processus de modernisation de concert avec celui de l'État, Diane Lamoureux demeure malgré tout l'une des premières au Québec à aborder ce genre de question.

Jacinthe Michaud

École d'Études des femmes

Université York (Canada)

\section{Jaap Lintvelt}

Aspects de la narration.

Thématique, idéologie et identité.

Québec, Nota bene, coll. "Littérature(s)"

et Paris, L'Harmattan, 2001.

\section{Jaap Lintvelt, Richard Saint-Gelais,}

Will Verhoeven et Catherine Raffi-Béroud [éd.]

Roman contemporain et identité culturelle

en Amérique du Nord. Contemporary fiction

and cultural identity in North America.

Québec, Nota bene, 1998.

Un colloque tenu en mai 1995 à l'Université de Groningue aux PaysBas qui s'intitulait a L'analyse de la représentation de l'identité culturelle dans le roman nord-américain depuis 1960* a donné lieu à ce collectif, qui regroupe vingt études d'universitaires d'Europe et d'Amérique (dont la moitié des textes sont rédigés en langue anglaise). L'identité culturelle se trouve ici spécifiquement au centre des préoccupations, mais telle qu'inscrite dans l'œuvre littéraire canadienne, québécoise, américaine ou mexicaine. En raison de la multitude des interrogations, des approches, des corpus et des perspectives liés à l'identité culturelle en Amérique du Nord, il est impossible de laisser ici toute la place qui reviendrait à chacun des textes.

Dans son introduction, Richard St-Gelais convient du "chemin spiralé - et non circulaire - qui réunit le roman contemporain et l'identité 